\title{
Novel Structures and Superconductivity of Silane under Pressure
}

\author{
Miguel Martinez-Canales, ${ }^{1,2}$ Artem R. Oganov, ${ }^{3,4}$ Yanming Ma, ${ }^{5}$ Yan Yan, ${ }^{5}$ Andriy O. Lyakhov, ${ }^{3}$ and Aitor Bergara ${ }^{1,2,6}$ \\ ${ }^{1}$ Materia Kondentsatuaren Fisika Saila, Zientzia eta Teknologia Fakultatea, Euskal Herriko Unibertsitatea, \\ 644 Postakutxatila, 48080 Bilbo, Basque Country, Spain \\ ${ }^{2}$ Donostia International Physics Center (DIPC), Paseo de Manuel Lardizabal, 20018, Donostia, Basque Country, Spain \\ ${ }^{3}$ Department of Geosciences and New York Center for Computational Science, State University of New York at Stony Brook, \\ Stony Brook, New York 11794-2100, USA \\ ${ }^{4}$ Geology Department, Moscow State University, 119992 Moscow, Russia \\ ${ }^{5}$ National Laboratory of Superhard Materials, Jilin University, Changchun 130012, People's Republic of China \\ ${ }^{6}$ Centro de Fisica de Materiales CSIC-UPV/EHU, 1072 Posta kutxatila, E-20080 Donostia, Basque Country, Spain
}

(Received 16 September 2008; published 27 February 2009)

\begin{abstract}
Following the suggestion that hydrogen-rich compounds, and, in particular, silane $\left(\mathrm{SiH}_{4}\right)$, might be high- $T_{c}$ superconductors at moderate pressures, very recent experiments have confirmed that silane metallises and even becomes superconducting at high pressure. In this article, we present a structural characterization of compressed silane obtained with an ab initio evolutionary algorithm for crystal structure prediction. Besides the earlier molecular and chainlike structures of $P 2_{1} / c$ and $I 4_{1} / a$ symmetries, respectively, we propose two novel structures with space groups $F d d 2$ and $P b c n$, to be stable at 25-55 and 220-250 GPa, respectively. According to our calculations, silane becomes metallic and superconducting at $220 \mathrm{GPa}$ in the layered $\mathrm{Pbcn}$ structure, with a theoretical $T_{c}$ of $16 \mathrm{~K}$. Our calculations also show that the imaginary phonons of the recently proposed $P 6_{3}$ generate the $P b c n$ structure.
\end{abstract}

DOI: 10.1103/PhysRevLett.102.087005

Soon after the BCS theory for superconductivity was established, the race towards room-temperature superconductivity started. Applying the BCS theory to hypothetic metallic hydrogen, Ashcroft realized that a very high- $T_{c}$ was plausible [1], placed by current precise calculations in $240 \mathrm{~K}$ at $400 \mathrm{GPa}$ [2]. Nevertheless, 70 years after metallic hydrogen was predicted [3], it still is an elusive goal of physics [4].

The quest for metallic and superconducting hydrogen got renewed interest with the discovery of the relatively high superconducting $T_{c}$ in lithium [5] under pressure. Furthermore, it has been recently suggested that hydrogen-rich compounds should retain a high- $T_{c}$ while lowering the threshold pressure for metallization, due to chemical precompression caused by heavier elements [6]. Group IV hydrides are obvious candidates, as according to the Goldhammer-Herzfeld criterion these compounds should metallize at moderate pressures. Theoretical [7] and experimental [8] work on methane has been so far unsuccessful, while theoretical predictions in germane [9] and stannane [10] have been more fruitful. However, silane has been the subject of most theoretical and experimental research so far.

There has been an ongoing theoretical discussion on the metallic transition of silane below $100 \mathrm{GPa}[11,12]$, and even some predictions on $T_{c}$ have emerged [11,13]. Although silane is a molecular crystal and an insulator up to at least $25 \mathrm{GPa}$ [14], recent experiments have found that silane forms a metastable metallic and superconducting phase above $60 \mathrm{GPa}$ [15], with a maximum $T_{c}$ of $17.5 \mathrm{~K}$ [16]. However, the superconducting structure reported, $P 6_{3}$, is unlike any other previously predicted [11-13].
PACS numbers: 74.70.Ad, 74.10.+v, 74.25.Jb, 74.62.Fj

The breakthrough of superconductivity in a hydride, together with the discrepancy of previous theoretical works with the experimental results, has prompted us to thoroughly analyze compressed silane using ab initio methods coupled with evolutionary structure prediction algorithms.

Stable and most competitive metastable structures of silane under pressure have been obtained with the $a b$ initio evolutionary algorithm USPEX [17-19]. This approach has been successfully used in the study of other materials at high pressure, e.g., $[9,20,21]$. The underlying $a b$ initio structural relaxations have been performed using density functional theory (DFT) [22,23] using the PBE exchange-correlation functional [24] as implemented in the VASP code [25]. Raman spectra, lattice dynamics, and electron-phonon coupling have been computed with QUANTUM-ESPRESSO [26]. The pressures and compact structures studied here do encourage the use of the allelectron PAW method [27].

As silane has been seen to evolve from a molecular crystal to polymeric chains under pressure, we have performed structure prediction simulations at $40,70,150$, and $300 \mathrm{GPa}$, with 1, 2, 3, 4, and $6 \mathrm{SiH}_{4}$ molecules per unit cell. In our evolutionary simulations, each generation consisted of 20-70 structures (increasing with system size), the first generation being random. Each subsequent generation was produced from the lowest-enthalpy $60 \%$ structures of the preceding generation; in addition, the lowest-enthalpy structure always survived into the next generation. The variation operators used for producing offspring included heredity (60\% structures), lattice mutation (30\%), and atomic permutation (10\%). Structural relaxations and enthalpy calculations were done with Monkhorst-Pack 
meshes with resolution better than $2 \pi \times 0.06 \AA^{-1}$. The most stable structures at these pressures have been studied further with increased accuracy between ambient pressure and $250 \mathrm{GPa}$. Grids denser than $2 \pi \times 0.025 \AA^{-1}$ and a $600 \mathrm{eV}$ energy cutoff have been used to perform the more accurate calculations.

In the lower pressure range, between 10 and $25 \mathrm{GPa}$, the most stable structure of those analyzed is the experimental one, with symmetry $P 2_{1} / c$ [14]. At $25 \mathrm{GPa}$, we have a pressure-induced structural transition to a phase of symmetry $F d d 2$ (depicted in Fig. 1), which is thermodynamically favored over the $P 2 / c$ structure, predicted using random sampling [12] for this pressure range, and remains stable up to $55 \mathrm{GPa}$. Actually, at $26.5 \mathrm{GPa}$, both x-ray [14] and Raman spectra [15] have identified a phase transition to an unidentified structure that up to $32 \mathrm{GPa}$ coexists with the $P 2_{1} / c$, which is in agreement with the $F d d 2$ phase being stable above $25 \mathrm{GPa}$. From the electronic point of view, the band gap [28] has a drastic reduction at the phase boundary, and in the $F d d 2$ structure, the gap keeps decreasing from 2 to $1 \mathrm{eV}$ in its pressure range of stability. The presence of such small gap might explain the strong changes in the optical properties at 27-30 GPa observed by Chen et al. [15], where silane begins to become black until the entire sample was observed to turn completely opaque, but not metallic, at $31.6 \mathrm{GPa}$. Parameters describing the orthorombic $F d d 2$ structure are listed in Table I. The silicon atoms occupy the 8a orbit, while hydrogen lies in two different $16 \mathrm{~b}$ orbits. This arrangement has $\mathrm{Si}$ atoms in the sixfold (distorted octahedral) coordination, with two $\mathrm{H}$ atoms at $1.46 \AA$, two at $1.57 \AA$, and two at $1.63 \AA$, so that the average $\mathrm{Si}-\mathrm{H}$ bond length is longer than the $\mathrm{Si}-\mathrm{H}$ distance in the free molecule, $1.47 \AA$. This is the first nonmolecular phase of silane. The minimum $\mathrm{H}-\mathrm{H}$ distance is $1.66 \AA$. The fact that the shortest $\mathrm{Si}-\mathrm{H}$ distance is similar to the $\mathrm{Si}-\mathrm{H}$ distance in the free molecule explains why from experimental Raman spectra [15] it was concluded that silane remains molecular in the pressure range 25$55 \mathrm{GPa}$. Actually, the presence of such short Si-H distances allows one to consider the $F d d 2$ phase as still displaying a partially molecular character. Further confidence in this structure is gained by examining its Raman spectrum. Our calculations reproduce the extremely large peak seen at $2200 \mathrm{~cm}^{-1}$, as well as the two smaller surrounding peaks, in [15]. We also predict a strong peak at $1400 \mathrm{~cm}^{-1}$, not shown in [15] but characteristic of the spectrum shown in [16]. The rest of the Raman intensities are much smaller. Our calculations also reproduce the small peaks at $1100 \mathrm{~cm}^{-1}$, but similar predicted peaks at $800 \mathrm{~cm}^{-1}$ are not seen experimentally. Lattice dynamics calculations also confirm the stability of this phase.

As shown in Fig. 2, at $55 \mathrm{GPa}$ there are four competitive structures $\left(F d d 2, I 4_{1} / a m d, P 2 / c\right.$ and $\left.I 4_{1} / a\right)$ with the same enthalpy. It must be noted that the latter three structures share the presence of $\mathrm{Si}-\mathrm{H}-\mathrm{Si}$ chains and are radically different from the semimolecular $F d d 2$. Above $55 \mathrm{GPa}$,
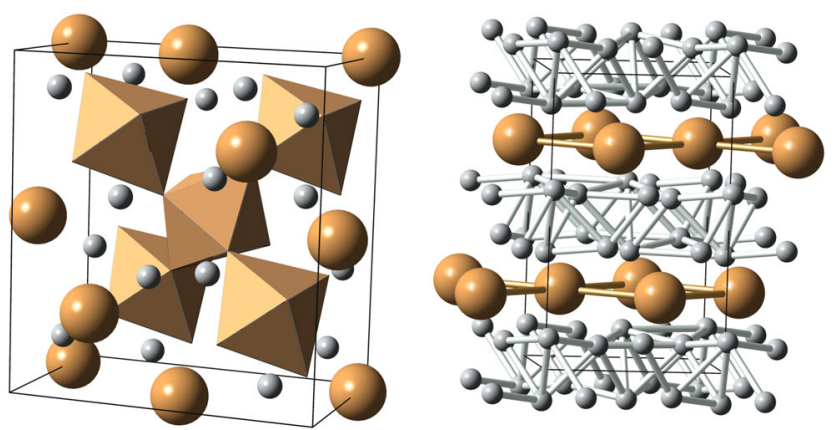

FIG. 1 (color online). Novel structures of silane. Large atoms depict $\mathrm{Si}$, while small atoms represent $\mathrm{H}$. Left: $F d d 2$ silane, stable between 25 and $55 \mathrm{GPa}$. Right: Pbcn silane, which is metallic, superconducting and favored above $220 \mathrm{GPa}$.

the $F d d 2$ structure undergoes a significant change, as the second neighbors of $\mathrm{H}$ atoms are no longer $\mathrm{H}$ but $\mathrm{Si}$ and, therefore, increasing the pressure will not just compress the structure but favors the formation of polymeric $\mathrm{Si}-\mathrm{H}-\mathrm{Si}$ chains and makes unavoidable the transition to the $I 4_{1} / a$ phase [12,16], which remains stable up to $220 \mathrm{GPa}$. Furthermore, although the $F d d 2$ structure is no longer preferred at higher pressure, above $70 \mathrm{GPa}$, it also undergoes significant changes and no longer displays any characteristic of a molecular crystal, but confirms the tendency to polymerization. This structural change implies a drastic reduction of the gap to around $0.65 \mathrm{eV}$ at $55 \mathrm{GPa}$, which remains constant at just above $0.5 \mathrm{eV}$ from 50 to almost $220 \mathrm{GPa}$. We recall that experiments $[15,16]$ observe metallization in a metastable phase at much lower pressures. Interestingly, although most available low-enthalpy candidates at around $70 \mathrm{GPa}\left(P 2 / c, I 4_{1} / a, I 4_{1} /\right.$ amd,$C 2 / c$, $P b c n)$ are no longer molecular and the arrangement of $\mathrm{H}$ atoms in the $\mathrm{Si}$ sublattice shows a preference for more

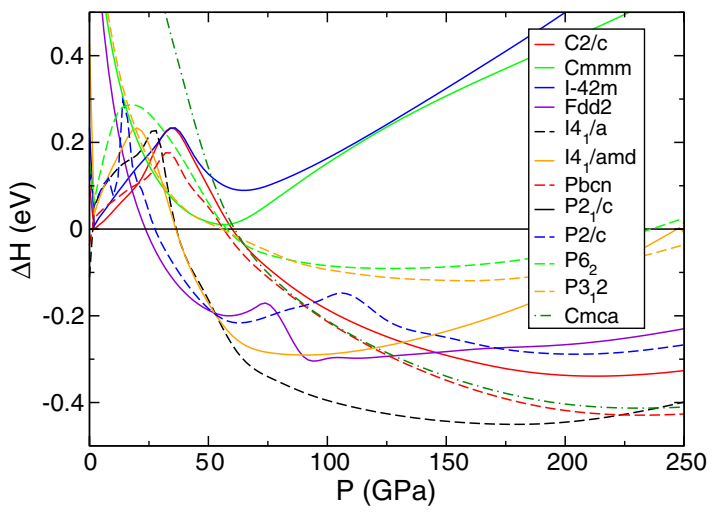

FIG. 2 (color online). Enthalpies of the most favorable structures. Interestingly, at $55 \mathrm{GPa}$, four competing structures have the same enthalpy and silane changes from intermediate between molecular and polymeric to a fully polymeric phase. The $P 6_{3}$ structure lies always at least $1 \mathrm{eV}$ above the reference $P 2_{1} / c$ structure. Lines are only a guide to the eye, the actual enthalpies being calculated at discrete intervals. 
TABLE I. Details of the new favored structures obtained in this work. The parameters of the $F d d 2$ structure correspond to 42 GPa, while the ones for $P b c n$ silane were obtained at $220 \mathrm{GPa}$.

\begin{tabular}{|c|c|c|c|c|c|}
\hline \multirow{2}{*}{$\begin{array}{l}\text { Space group } \\
F d d 2\end{array}$} & \multirow{2}{*}{$\begin{array}{c}\text { Lattice parameters }(\AA) \\
a=6.567\end{array}$} & \multicolumn{4}{|c|}{ Atomic coordinates (fractional) } \\
\hline & & $\mathrm{Si}$ & 0.0 & 0.0 & 0.6126 \\
\hline & $b=7.075$ & H1 & 0.1441 & 0.0993 & 0.3994 \\
\hline & $c=4.063$ & $\mathrm{H} 2$ & 0.8615 & 0.1745 & 0.6960 \\
\hline \multirow[t]{3}{*}{$P b c n$} & $a=3.198$ & $\mathrm{Si}$ & 0.5 & 0.8765 & 0.25 \\
\hline & $b=3.177$ & H1 & 0.8431 & 0.6946 & 0.0718 \\
\hline & $c=5.462$ & $\mathrm{H} 2$ & 0.1262 & 0.9997 & 0.6091 \\
\hline
\end{tabular}

compact and polymeric-like structures, all the thermodynamically relevant structures are insulators. This changes radically at $150 \mathrm{GPa}$. At this pressure, most competitive structures display a metallic alloy character instead of a polymeric-like, with two clear exceptions: the $I 4_{1} /$ amd structure, which can be described as hydrogen tetrahedra sandwiched between silicon layers, and the $I 4_{1} / a$ structure displaying the double Si-H-Si chains. Both of these structures remain insulating and the gap of the preferred structure also remains almost constant in this pressure range, in agreement with previous calculations [12].

On the other hand, the $P 6_{3}$ structure [16] proposed for the superconducting silane from $\mathrm{x}$-ray measurements between 50 and $150 \mathrm{GPa}$, significantly deviates from the structures analyzed above and, although our calculations confirmed that it is metallic in the whole pressure range studied, is plagued with issues that place heavy doubts on its feasibility. Its enthalpy is always more than $1 \mathrm{eV} /$ molecule higher than that of the reference $P 2_{1} / c$ structure in the whole pressure range and, according to our calculations, the experimental atomic positions and lattice parameters are far from local equilibrium, with very large forces on the atoms (over $1 \mathrm{eV} / \AA$ ). Furthermore, the stress tensor computed at fixed structure is strongly nonhydrostatic $\left(\sigma_{z z}-\sigma_{x x} \approx 10 \mathrm{GPa}\right)$ and corresponds to much higher pressure $(220 \mathrm{GPa})$ than the experimental one (120 GPa in [16]). As seen in Fig. 3, the experimental EOS is in very good agreement with the theoretical one as long as the insulator path is taken $\left(P 2_{1} / c, F d d 2\right.$ and $\left.I 4_{1} / a\right)$ but the agreement with the EOS determined in [16] for the metallic phase is extremely poor - even the EOS of the $P 6_{3}$ structure of [16], when relaxed, deviates from the EOS proposed in [16]. This difference in volume is much larger than the typical DFT error. It must be pointed out that none of the competitive structures found in this pressure range, including $P 6_{2}$ and $P 3_{1} 2$, are able to reproduce the experimental diffraction pattern observed in [16]. So although the proposed $P 6_{3}$ structure is dynamically unstable and relaxes into the $P b c n$ structure proposed here as stable over $220 \mathrm{GPa}$, we believe a more profound difference exists at around $100 \mathrm{GPa}$. Given the difficulty to locate hydrogen atoms, it is possible that both the experimental EOS and diffraction pattern might be even related to a possible partial dissociation of silane due to a reconstructive phase transition occurring at around $55 \mathrm{GPa}$.

We believe that the key factor of this anomalous behavior observed in silane is located at $55 \mathrm{GPa}$, where the enthalpies of many competitive structures converge (Fig. 2). Analyzing the evolution with pressure of the distance to the third neighbor allows us to conclude that silane is changing from partly molecular to fully polymeric at $55 \mathrm{GPa}$, and suffers a thorough change. The energetically favored transition $F d d 2 \rightarrow I 4_{1} / a$ is reconstructive, and may involve a high activation barrier. At room temperature (used in experiments reported in [16]), a loweractivation path is followed instead, leading to a metallic metastable phase that might retain some structural similarity with the $F d d 2$ phase.

In the high pressure range, as seen in Fig. 2, insulating tetragonal $I 4_{1} / a$ eventually gives way to a new metallic structure. Although the $C 2 / c$ structure found here differs from the previous one [12] in a rearrangement of two of the hydrogen orbits into a general orbit, making it slightly more favorable, a new structure of symmetry $P b c n$ is preferred. This new structure becomes favorable at $220 \mathrm{GPa}$, marking the threshold at which, according to our calculations, silane adopts a metallic ground state. This

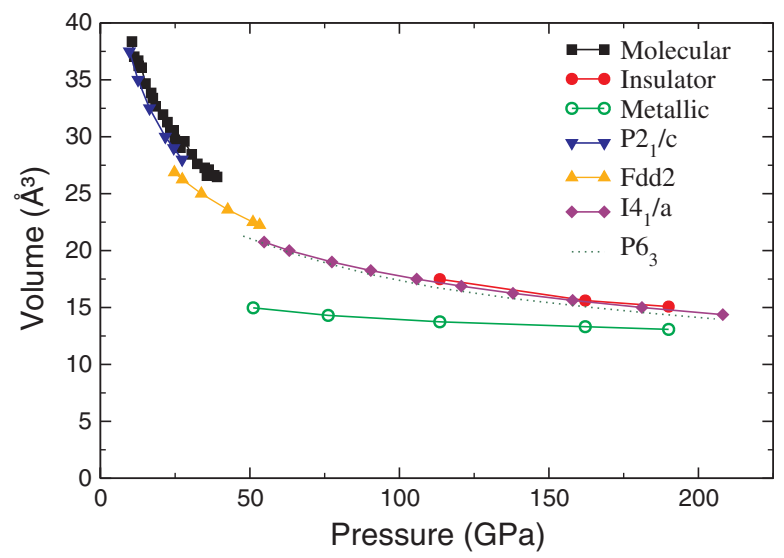

FIG. 3 (color online). Theoretical equation of state (EOS) of silane, compared with the experimental one [16]. Also included is the equation of state corresponding to the relaxed $P 6_{3}$ structure. Note the discrepancy between the EOS of metallic silane and the calculations for all other phases. 


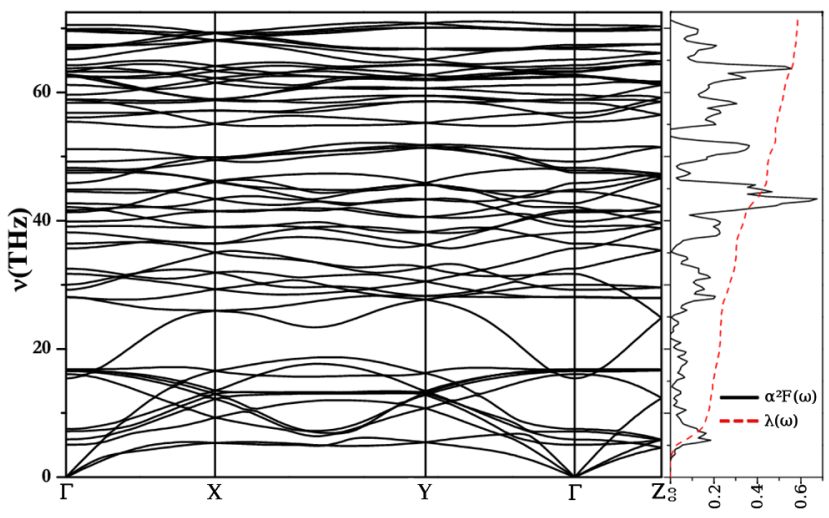

FIG. 4 (color online). Phonon dispersion and spectral Eliashberg function of the Pbcn structure at $190 \mathrm{GPa}$. This pressure has been chosen to show a better comparison to experiment [16]. At $190 \mathrm{GPa}, P b c n$ silane has a $T_{c}=16.5 \mathrm{~K}$, in nice agreement with the experimental value $\left(T_{c}=12 \mathrm{~K}\right)$.

structure is not only more stable than Cmca silane [29], but can also arise from the dynamic instabilities of the proposed $\mathrm{Pb}_{3}$ structure. The $\mathrm{Pbcn}$ structure shows an interlayer alloylike atomic arrangement, with slightly displaced almost square $\mathrm{Si}$ layers and $\mathrm{H}$ atoms lying in the interlayer spaces of the structure. Regarding the atomic distances, even at $220 \mathrm{GPa}$, the closest $\mathrm{H}-\mathrm{H}$ distance is $1.35 \AA$, far from the dimer length $(0.74 \AA)$. Hinting the possibility of a metallic alloy, it is very similar to the $\mathrm{Si}-\mathrm{H}$ distance, this being $1.475 \AA$. Given the layered character of the Pbcn structure and the high concentration of $\mathrm{H}$ atoms, it is likely that this structure might display electronic similarities to pure metallic hydrogen not seen in previously proposed structures. To better compare with experiments, the electron-phonon calculations have been performed at $190 \mathrm{GPa}$. The phonons and the spectral function are depicted in Fig. 4. The resulting value of $\lambda$ is 0.58 . This value is not very high, but the high $\omega_{\log }$ caused by the hydrogen vibrons helps yield a $T_{c}$ of $16.5 \mathrm{~K}$ after using a typical value of $\mu^{\star}=0.13$, according to the Allen-Dynes formula [30]. This is in very good agreement with the experimental temperature of $12 \mathrm{~K}$ recorded at that pressure. The use of the evolutionary algorithm USPEX [17-19] allowed us to find lower enthalpy structures than those found in previous studies where chemical intuition [11,29] or a simple random sampling search [12] were used for structure search.

The authors acknowledge funding from the Spanish Ministry of Education (Grants No. BFM2003-04428 and No. BES-2005-8057) and Swiss National Science Foundation (Grants No. 200021-111847/1 and No. 200021116219). USPEX calculations were performed at the Swiss National Supercomputer Centre, on Skif MSU supercomputer at Moscow State University, and at the Joint Supercomputer Centre of the Russian Academy of Sciences. We thank SGIker Arina UPV for the allocation of computational resources, which were used for analyzing energetic and electronic properties of silane phases. The authors are also thankful to the Bilbao Crystallographic Server [31], whose useful tools have been thoroughly used for this research.

[1] N. W. Ashcroft, Phys. Rev. Lett. 21, 1748 (1968).

[2] P. Cudazzo et al., Phys. Rev. Lett. 100, 257001 (2008).

[3] E. Wigner and H. Huntington, J. Chem. Phys. 3, 764 (1935).

[4] P. Loubeyre, F. Occelli, and R. LeToullec, Nature (London) 416, 613 (2002).

[5] K. Shimizu, H. Ishikawa, D. Takao, T. Yagi, and K. Amaya, Nature (London) 419, 597 (2002).

[6] N. W. Ashcroft, Phys. Rev. Lett. 92, 187002 (2004).

[7] M. Martinez-Canales and A. Bergara, High Press. Res. 26, 369 (2006).

[8] L. Sun, A. L. Ruoff, C. S. Zha, and G. Stupian, J. Phys. Chem. Solids 67, 2603 (2006).

[9] G. Gao, A. R. Oganov, A. Bergara, M. Martinez-Canalez, T. Cui, T. Iitaka, Y. Ma, and G. Zou, Phys. Rev. Lett. 101, 107002 (2008).

[10] J. S. Tse, Y. Yao, and K. Tanaka, Phys. Rev. Lett. 98, 117004 (2007).

[11] J. Feng, W. Grochala, T. Jaroń, R. Hoffmann, A. Bergara, and N. W. Ashcroft, Phys. Rev. Lett. 96, 017006 (2006).

[12] C. J. Pickard and R. J. Needs, Phys. Rev. Lett. 97, 045504 (2006).

[13] Y. Yao, J. S. Tse, Y. Ma, and K. Tanaka, Europhys. Lett. 78, 37003 (2007).

[14] O. Degtyareva et al., Phys. Rev. B 76, 064123 (2007).

[15] X.-J. Chen et al., Proc. Natl. Acad. Sci. U.S.A. 105, 20 (2008).

[16] M. I. Eremets, I. A. Trojan, S. A. Medvedev, J. S. Tse, and Y. Yao, Science 319, 1506 (2008).

[17] A. R. Oganov and C.W. Glass, J. Chem. Phys. 124, 244704 (2006).

[18] A. R. Oganov, C. W. Glass, and S. Ono, Earth Planet. Sci. Lett. 241, 95 (2006).

[19] C. W. Glass, A. R. Oganov, and N. Hansen, Comput. Phys. Commun. 175, 713 (2006).

[20] Y. Ma, A. R. Oganov, and Y. Xie, Phys. Rev. B 78, 014102 (2008).

[21] Y. Ma, A. R. Oganov, and C. W. Glass, Phys. Rev. B 76, 064101 (2007).

[22] P. Hohenberg and W. Kohn, Phys. Rev. 136, B864 (1964).

[23] W. Kohn and L. J. Sham, Phys. Rev. 140, A1133 (1965).

[24] J. P. Perdew, K. Burke, and M. Ernzerhof, Phys. Rev. Lett. 77, 3865 (1996).

[25] G. Kresse and J. Furthmuller, Phys. Rev. B 54, 11169 (1996).

[26] S. Baroni et al., http://www.pwscf.org/.

[27] P. E. Blöchl, Phys. Rev. B 50, 17953 (1994).

[28] A figure depicting the evolution of the band-gap with pressure in silane is provided as supplementary material. See EPAPS Document No. E-PRLTAO-102-016910. For more information on EPAPS, see http://www.aip.org/ pubservs/epaps.html.

[29] X.-J. Chen et al., Phys. Rev. Lett. 101, 077002 (2008).

[30] R. C. Dynes, Solid State Commun. 10, 615 (1972).

[31] M. I. Aroyo et al., Z. Kristallogr. 221, 15 (2006). 